\title{
Invasion and egress cascade in intracellular protozoa: Part 1
}

\author{
Editorial Sherif M Abaza
}

Medical Parasitology Department, Faculty of Medicine, Suez Canal University, Ismailia, Egypt

A wide spectrum of pathogenic intracellular bacteria, viruses and protozoa adapt several strategies to enter into and exit from their host with optimum rates of survival, replication, and progression through life cycle stages as well as transmission. Egress is defined as the escape process from the host cell after replication and is followed by daughter cells exit. Therefore, egress is of fundamental importance for transmission processes, pathogen spread, and inflammation. Accordingly, molecules involved in egress mechanism(s) act as key factors in infection, pathogenesis, and transmission. They are considered as indirect virulence factors and potential drug targets. In fact, the egress cascade is a complex strategy involving several aspects, and its molecular analysis is difficult to investigate in vitro due to the contribution of several molecules in invasion or egress. It is impossible to independently investigate factors involved in egress from vacuolar and host cell membranes at the same time. The present editorial aims to simplify our knowledge regarding this critical issue with emphasis on its molecular tools and mechanisms.

Keywords: apical secretory organelles, drug targets, egress, invasion, Plasmodium, protein kinases, Toxoplasma.

Received: 12 March, 2021, Accepted: 16 April, 2021.

Corresponding Author: Sherif M Abaza, Tel.: +20 1005243428, E-mail: smabaza@hotmail.com

Print ISSN: 1687-7942, Online ISSN: 2090-2646, Vol. 14, No. 1, April, 2021.

One of the major characters of intracellular pathogens is the considerable difference in host cell preference. Accordingly, they depend on specific requirements and establish suitable mechanisms for cell entry, modes of replication, and cell exit. The main obstacle in invading a specific target host cell is crossing the cellular plasma membrane and cytoskeleton. Several strategies are described that differ according to parasite' capabilities and nature of the invaded host cells. In intracellular apicomplexans, Plasmodium spp. and T. gondii utilize the characteristic secretory apical organelles that release molecules through a moving junction to actively drive the parasite into host cells. Molecules released from micronemes are the key factors for strong adhesion of apicomplexans to the host cell surface, while rhoptry proteins are responsible for formation of a tight moving junction. The formed junction moves through the host cell plasma membrane to alter its actin cytoskeleton. It is worth mentioning that sporozoites of Cryptosporidium spp. do not actively invade host cells, instead the merozoites prompt host epithelial cells to surround them with a host cell-derived membrane at the tip of the epithelial cells under the brush border.

In intracellular hemoflagellates, Leishmania spp. not only utilize flagellar activity to invade host cells, but also once inside the host cell, the transformed amastigotes recruit phagocytosis of host cytosol to resist macrophages' phagocytic degradation. Host macrophage receptors mediate parasite adherence and subsequent phagocytosis signaling. On the other hand, T. cruzi recruit host cell lysosomes to the attachment site. Lysosomes fusion to host cell plasma membrane allows parasite internalization. Utilizing this strategy, T. cruzi ravages the endocytic pathway(s) used by the host cell to repair its plasma membrane. Leishmania promastiogotes showed their ability to utilize a similar strategy in host cell invasion in vitro. Besides, several surface lipid-anchored glycoproteins are established as virulence factors since all pathogenic trypanosomatids dramatically change composition of their glycocalyx membrane during stage transformation for host cell invasion. Sporulated intracellular protozoa (Microsporidium spp.) induce endocytosis utilizing their specialized polar tube proteins. Electron microscopy studies show that Microsporidium polar tube is surrounded by host cell plasma membrane.

Meanwhile, the process of cell entry, replication, and egress occurs in sequence, i.e., no egress without pathogen replication. The main obstacle that faces intracellular replication is the surrounding acidic environment produced as a result of fusion with endolysosomes. Therefore, all intracellular protozoa (except for T. cruzi) replicate in their parasitophorous vacuole (PV). While PV membrane is host-derived in Plasmodium spp., and T. gondii, other intracellular protozoa possess a parasite-derived PV membrane. Accordingly, egress in Plasmodium spp., and T. gondii requires breakdown of two barriers, namely $\mathrm{PV}$ and host cell membranes. To preserve host cell integrity, required for parasite replication, T. cruzi amastigotes breakdown the PV membrane to replicate in the host cytosol. To initiate egress, they have to breakdown host cell membrane. It is important to know that control of parasite egress by the host adaptive immune response leads to chronic infection.

In addition to membrane(s) lysis, active egress cascade includes disintegration of host cell cytoskeleton. Role of several molecules such as proteases, phospholipases, and pore-forming proteins are established in various intracellular protozoa. Such 
key molecules require activation factors such as calcium (Ca) concentration and/or its signaling pathway. Besides, egress cascade may utilize molecular mimicry to steal host cellular functions, e.g., host cytoskeleton dynamics. It is worth mentioning that egress cascade in few intracellular pathogens e.g., Cryptosporidium spp., does not result in host cell damage, most probably due to its presence in the brush border of the intestinal epithelium. Since membrane lysis and cellular damage triggers host inflammatory response with recruitment of immune cells, egress cascade in apicomplexans involves other strategies such as immunomodulation, autophagy and even apoptosis for the sake of the parasite survival.

Initial stimulus: Except for T. gondii (discussed later), stimuli to start host cell egress remains obscure for most intracellular pathogens. Mechanism utilized by intracellular pathogens to sense the environmental changes such as increase Ca concentration, or decrease host cell cytosolic potassium, are poorly understood. Although this is not essentially required for the process of egress cascade itself, sensing environmental changes is proposed as initial stimuli for egress in the majority of intracellular protozoa. This was observed microscopically (Azab ME personal communication) when bradyzoites in a tissue cyst became agitated by trypsinization of the cyst wall, and actively rolled over themselves, until one of them bore through the cyst wall by its apical complex, releasing the encysted bradyzoites.

\section{Tools}

1. Secretory organelles: Evolutionary studies showed that Superphylum Alveolata constitutes a major clade within Eukarya. Alveolates are a group of protists with cortical alveoli (sacs or cavities or vesicles), that are packed into a continuous layer beneath protists' membrane. Alveolata includes several free living and parasitic protozoa, among them ciliates, and apicomplexans. It was hypothesized that apicomplexan secretory organelles are evolutionary cavities adapted for several requirements of variable parasite lifestyles, i.e, nutrients acquisition, gliding motility, host cell exit, cytoadherence, and entry into a newly formed PV within another host cell. Secretory organelles include apical micronemes and rhoptries (Rhs), required for host cell invasion, as well as non-apical dense granules and osmiophilic bodies (OBs) required for the complete disruption of PV membrane.

- Micronemes: They are triggered by intracellular Ca-signaling pathway after host cell attachment. In Plasmodium spp., and T. gondii, micronemes release proteins on the parasite surface membrane. Among them are actinomyosin for motility, catalytic proteases, and perforin-like proteins (PLPS) for degradation of host cell membrane (discussed later). Micronemes number and released proteins differ according to apicomplexan life cycle stages. Plasmodium erythrocytic merozoites contain fewer micronemes than those of sporozoites, while Toxoplasma tachyzoites and Cryptosporidium sporozoites contain numerous micronemes. Toxoplasma micronemes are involved in host cell invasion through regulation of the contact between host receptors and parasite actin for gliding motility utilized in invasion strategy. Additional related secretory organelles such as exonemes and mononemes are identified in Plasmodium life cycle stages involved during invasion and egress cascade.

- Rhoptries: The apical membrane antigen (AMA1), released from micronemes was hypothesized to trigger release of $\mathrm{Rh}$ proteins. These pear or club-shaped organelles release their proteins after micronemes release theirs. There are two sets of proteins named according to localization of their production from neck or bulb regions. Neck proteins are responsible for stabilization of the tight moving junction between the parasite and the host cell, followed by bulb proteins. The latter carry out a broad spectrum of functions that include cell adhesion, PV formation in newly invaded host cells and immune evasion.

- Dense granules: They are organelles dispensed as microspheres, surrounded by a unique membrane. Immediately and constitutively after PV formation, they release dense granules proteins (GRAs) without a known molecular trigger. The GRAs are essentially responsible for formation of an intimate interaction with the host derived PV membrane inducing organization and proteins trafficking.

- Osmiophilic bodies (OBs): They are electron-dense organelles derived from Golgi vesicles in Plasmodium gametocytes. They first appear in stage IV female gametocytes, and occasionally in male gametocytes and increase in frequency and density with maturity to stage $\mathrm{V}$ gametocytes. They are involved in degradation of both PV membrane and RBC membrane skeleton, contributing to gamete egress in mosquitoes prior to gametogenesis. The PfG377 protein is the main component for OBs biogenesis. The OBs release two proteins into the PV that play a crucial role in gametocytes egress, and sporozoite traversal (GEST). Additionally, they constitute the protein of early gametocyte stage 3 (PEG3), also known as male development 1 (MDV1). Dynamics of OBs in $P$. falciparum activated gametocytes is investigated using antibodies raised against G377. It was demonstrated that OBs migrate to localize just beneath gametocyte' plasma membrane, and fuse together forming one vesicle. Prior to egress, the fused vesicle releases G377 into PV by exocytosis, i.e., membrane fusion of both PV and the fused vesicle. This OBs migration and fusion allows translocation of adhesins of thrombospondinrelated anonymous protein (TRAP) from micronemes to sporozoites' surface. Adhesins are essential for mature sporozoites migration from the mosquito hemocoel into the salivary gland lumen as well as in the skin following the mosquito bite and liver invasion. Surface-associated TRAP family proteins have an 
essential role in parasite motility and cell invasion because they provide the link between intracellular actomyosin motor and its extracellular substrate.

2. Pore-forming proteins: All intracellular apicomplexans PLPs, released from micronemes, are implicated in host cell traversal during invasion and egress. These proteins are members of the membrane attack complex/perforin (MACPF), a diverse family of pore forming proteins possessing a domain that binds with membrane' phospholipids receptors, the first step in pore formation. In human, members of MACPF superfamily play essential roles in immune response involving cytolysis of invading pathogens and infected cells. Plasmodium spp. possess five PLPs, whereas T. gondii expresses two PLPs. Among Plasmodium PLPs, only two are involved in egress of erythrocytic merozoites (PLP1) from host RBCs, and gametocytes (PLP2) in mosquito' midgut. The remaining PLPs (3-5) are incriminated in ookinetes and sporozoites cell traversal through midgut epithelium avoiding mosquito' immune defense. Another two molecules are uniquely expressed in Plasmodium spp. for cell traversal through host cells and mosquito's midgut. They are known as sporozoite protein essential for cell traversal (SPECT1) and ookinetes and sporozoites cell traversal (CelTOS), respectively (Figure 1).

3. Protein kinases: These are essentially incriminated in invasion and egress cascade. They mediate the transfer of phosphate groups from adenosine triphosphate (ATP) to specific residues on the target proteins, changing its stability and activity. Both Plasmodium spp. and T. gondii contain a family of Cadependent protein kinases (CDPKs) that have critical roles in the regulation of several processes including gliding motility, host cell attachment, invasion, and egress. It is established that T. gondii CDPK1 is crucial for protein secretion from the micronemes. Due to their peripheral localization, TgCDPK3 and PfCDPK5, molecules in Ca-dependent signaling pathway, are stimulated with decreased cellular potassium concentration because of cell permeabilization or damage. Additionally, proteomic analysis of $P$. falciparum revealed 21 members of unique protein kinases (FIKK family). Among them, only three FIKKs $(4.2,7.1$, and 12) are exported to the newly infected $\mathrm{RBC}$ to alter its membrane rigidity. Due to its restricted localization in Rhs, PfFIKK3, is not exported into RBCs; its role in the release of $\mathrm{Rh}$ bulb proteins during $\mathrm{RBC}$ invasion is hypothesized.

\section{Invasion and egress in intracellular protozoa Plasmodium spp.}

Molecular studies in Plasmodium spp. confirmed that RBCs invasion and egress are tightly regulated by a Plasmodium protease arsenal. This includes subtilisinlike serine proteases (SUBs 1 and 2), serine repeats antigens (SERAs 5 and 6), as well as falcipians (Fals 1 and 2), and dipeptidyl aminopeptidase 3 (DPAP3) as cysteine proteases, and plasmepsins (Plas IX and X) as aspartyl proteases.

Host cell invasion: SUB1 contributes with DPAP3 in processing of merozoite surface protein 1 (MSP1) and the specific rhomboid protease 1 (ROM1), whereas SUB2 is required for processing of proteases (Fal1 and PlaIX). Mature SUB2 regulates MSPs release involved in shedding of merozoites protein coat, a crucial step for attachment to RBC surface during the invasion process. It is worth mentioning that MSPs are synthesized in early schizogony and MSP1 is the first molecule that attaches to a new RBC surface, targeting spectrin, a complex on the internal surface of RBC cell membrane. It was observed that MSP1 serves as a marker for the formation of merozoites food vacuoles. In addition, ROM1 is involved in PV formation, and cleavage of intramembrane merozoite adhesins. Its crucial role in de novo invasion is hypothesized due to its apical localization in egressed merozoites. Besides, $P$. falciparum unique protein kinase (PfFIKK3) triggers $\mathrm{Rh}$ bulb proteins release. While Fal1 utilizes its proteolytic activity in degradation of RBC plasma membrane, PlaIX that is localized in Rhs, is expressed for continuous maturation of $\mathrm{Rh}$ proteins in invasive merozites (Figure 1, and table 1).

Hepatic merozoites egress: Liver-specific protein 1 (LISP1) contributes with proteases in merozoites egress from hepatocytes. It is expressed by hepatic merozoites to localize at $\mathrm{PV}$ membrane. In vitro studies showed that merozoites deficient in LISP1 developed well into hepatic merozoites with normal RBCs infectivity. However, in vivo studies showed that hepatic merozoites deficient in LISP1 were not able to rupture PV membrane, i.e., they are trapped inside hepatocytes, with low levels of parasitemia in infected mice. Besides, merozoites egress utilizes a budding mechanism to release intra vesicular merosomes, from hepatic cytosol into hepatic sinusoids. Merosomes membranes are derived from the infected hepatocyte to protect egressed merozoites. Finally, merozoites are released in the pulmonary microvasculature (Figure 1, and table 1).

Erythrocytic merozoites egress: As previously mentioned, merozoite egress through PV membrane is firmly controlled by proteases. Other effectors are reported for rapid lysis succession of RBCs plasma membrane, and cleavage of RBCs cytoskeletal elements such as phospholipases, PLPs and osmotic stress proteins. Protein kinases unique in $P$. falciparum (PfFIKKs 4.2, 7.1 and 12) alter RBC membrane rigidity. Plasmodium SUB1 possesses unique evolutionary structural feature in utilizing specific Ca-dependent regulation of its activation to initiate egress. After a series of proteolytic processing, mature SUB1 is stored in merozoites exonemes. Due to increased intracellular Ca concentration, exonemes release SUB1 in PV to contribute with DPAP3 in processing SERAs 5 and 6. 
The latter proteases play a crucial role in the proper rupture of PV membrane and destabilization of RBC cytoskeleton. While Fal2 is involved in degradation of erythrocyte-membrane skeletal proteins including ankyrin, PlaX is expressed in mature schizonts to control SUB1 maturation. It is observed that PlaX has a crucial role for the final SUB1 processing to the degree that it is described as the most upstream protease in the egress. Merozoites egress is a rapid process $(\sim 400$ milli-sec) that takes place in three main steps: 1) a single $\sim 1 \mu \mathrm{m}$ pore that allows osmotically-driven release of 1-2 merozoites; 2) an outward curling or wrinkling of $\mathrm{RBC}$ membrane from the pore center, followed by 3) a rapid eversion or buckling of the entire membrane to eject all merozites, i.e., vesiculation. Prior modification and/or degradation of RBC cytoskeleton facilitates the last two steps (Figure 1, and table 1).

Gametocytes egress: It is demonstrated in four steps: 1) swelling of host RBC; 2) PVM rupture and immediate vesiculation; 3) extrusion of these vesicles through a single pore in RBC membrane; and 4) RBC membrane is subsequently vesiculated releasing egressed gametes inside mosquito' midgut. It is worth mentioning that timing is critical $(30 \mathrm{~min})$ for female gametes fertilization with the short-lived male gametes. Egress is initiated by three stimuli: two external involving decreased temperature and excretion of xanthurenic acid from midgut, and one internal in the form of proteases activity. Predominantly in female gametocytes, OBs represent the secretory organelles with similar role as exonemes in erythrocytic stages. It is observed that G377 is exclusively expressed in females; while GEST, PEG3 and PPLP2 are expressed in both sexes. Proteomic studies identified four patatinlike phospholipases (PATPLs) with lipolytic activity in $P$. falciparum gametocytes. It is observed that PfPATPL1 plays an essential role in PLP2 translocation at gametes periphery (micronemes) to promote egress in mosquito' midgut. In addition, Plasmodium spp. express a TRAP family protein termed merozoite-TRAP (MTRAP) that was thought to be essentially involved in RBCs invasion by merozoites. Nowadays, PfMTRAP identified in TRAPcontaining secretory vesicles, proved to have an essential role in gamete egress from RBCs in mosquito' midgut. It contributes to disruption of PV and RBC membranes (Figure 1 and table 1).

Sporozoites egress in mosquitoes: Besides its role in gametocytes egress, GEST contributes with a SERA homologue, known as egress cysteine protease 1 (ECP1), in successful exit of sporozoites from oocyst. Previously,

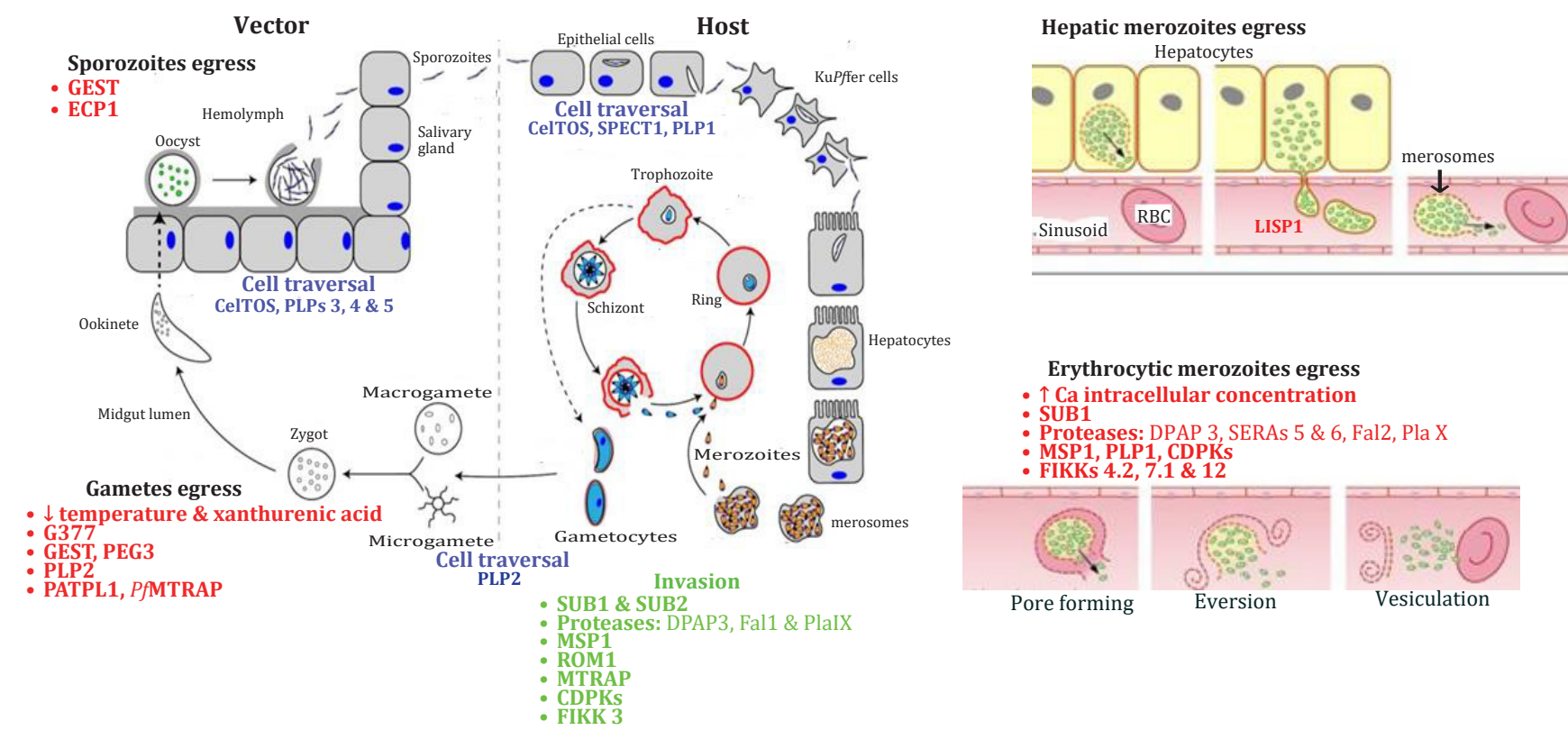

Fig. 1. Molecules for invasion (green), egress (red) and cell traversal (blue) of different stages in Plasmodium spp. life cycle. Modified from figures cited by Friedrich et al. ${ }^{[3]}$ and Guerra and Carruthers ${ }^{[5]}$.

In host cell invasion, SUB1 contributes with DPAP3 in processing MSP1 (protein coat shedding) and ROM1 (ahesins cleavage). SUB2 processes Fal1 (RBC plasma membrane disruption) and PlaIX (maturation of rhoptry proteins). MTRAP promotes merozoites motility, FIKKs regulate release of rhoptry proteins, and alter RBC membrane rigidity. CDPKs regulate protein phosphorylation required for several processes. Hepatic merozoites egress involves PV membrane lysis, release of merozoites in merosomes (LISP1), and merosome lysis. Erythrocytic merozoites egress includes PV membrane lysis, and breakdown of RBCs cytoskeletal elements by pore forming proteins (PLP1), MSP1 and proteases. RBCs undergo sequent events ending with merzoites vesiculation. For gametes egress, G377 is involved in biogenesis of serectory organelles, osmiophilic bodies release GEST and PEG3, PfPATPL1 translocates PLPs, and PfMTRAP disrupts PV and RBC membranes. GEST contributes with ECP1 for sporozoites egress.

Ca: Calcium; CelTOS: Ookinetes and sporozoites cell traversal; DPAP3: Dipeptidyl aminopeptidase 3; ECP1: Egress cysteine protease 1; Fal: Falcipian; G377: Gametocytes protein; GEST: Gamete egress and sporozoite traversal protein; LISP: Liver specific protein; MSP: Merozoite surface protein; MTRAP: Merozoite thrombospondin-related anonymous protein; PARPL1: Patatin-like phospholipase; PEG3: Protein of early gametocyte 3, Pla: Plasmepsin; SERA: Serine repeat antigen; SPECT: Sporozoite protein essential for cell traversal; SUB: Subtilisin-like serine protease. 
it was thought that sporozoites exit oocysts through passive rupture due to parasite growth. However, it was proved that ECP1 is expressed in mature oocysts, and subsequently downregulated in the mosquito's salivary gland sporozoites before transmission to the mammalian host. Knocking out the Plasmodium's encoding gene blocks its life cycle at the oocyst stage (Figure 1 and table 1).

\section{CONCLUDING REMARKS}

1. Intracellular protozoa utilize different strategies for host cell invasion; tight moving junction (Plasmodium spp. and T. gondii), recruitment of either host cell phagocytosis (Leishmania spp.) or host cell lysosomes (T. cruzi), and induction of endocytosis for spores internalization (Microsporidium spp.) Cryptosporidium spp. do not actively invade host cells.

2. In Plasmodium spp. and T. gondii, replication occurs within PV followed by breakdown of both membranes (PV and host cell). In pathogenic kinetoplastids (except for T. cruzi), Cryptosporidium spp., and microsporidia, replication occurs within the host cell, followed by host cell membrane lysis. In T. cruzi, the first step is breakdown of its vacuole membrane, then replication inside host cytosole, followed by host cell membrane.

3. In Plasmodium spp. and T. gondii, a link exists between egress and subsequent de novo invasion that involves possession of apical secretory organelles (micronemes, rhoptries, and dense granules) as storage for the involved molecules, and Ca-signaling as initial stimuli. Besides, PLPs released from

Table 1. Plasmodium molecules involved in invasion and egress cascade.

\begin{tabular}{|c|c|c|c|}
\hline \multirow{2}{*}{ Molecule } & \multirow{2}{*}{ Localization } & \multicolumn{2}{|r|}{ Function } \\
\hline & & Invasion & Egress \\
\hline Ca & Intracellular & --- & Initiator \\
\hline CDPK & --- & \multicolumn{2}{|c|}{ Regulates protein phosphorylation required for host cell invasion and egress } \\
\hline DPAP3 & $\begin{array}{l}\text { Apical secretory } \\
\text { organelles }\end{array}$ & --- & Processing of SERAs 5 and 6 \\
\hline ECP1 & Mature oocyst & --- & Sporozites egress from oocyst \\
\hline Fal1 & PV & Proteolytic degradation & --- \\
\hline Fal2 & PV & --- & Degradation of RBC membrane skeletal proteins \\
\hline FIKK3 & Rhs & Release of Rh bulb proteins & --- \\
\hline FIKK4.2, $7.1 \& 12$ & Exported to RBCs cytosol & --- & Alter RBC membrane rigidity \\
\hline G377 & Female gametocyte Obs & --- & Biogenesis of secretory organelles \\
\hline GEST & Female gametocyte Obs & --- & Gametocytes and sporozoites egress \\
\hline LISP1 & Hepatocytes & --- & Hepatic merozoites egress \\
\hline MSPs & $\begin{array}{l}\text { Merozoite surface } \\
\text { membrane }\end{array}$ & $\begin{array}{l}\text { Shedding merozoites protein } \\
\text { coat }\end{array}$ & PV membrane disruption \\
\hline MTRAP & Micronemes & Merozoite motility & $\begin{array}{c}\text { Disruption of PV and RBC membranes in gametes } \\
\text { egress }\end{array}$ \\
\hline PATPL1 & --- & --- & PLP2 translocation at gametes periphery \\
\hline PEG3 & Male gametocyte Obs & --- & Gametocytes egress \\
\hline Pla IX & Rhs & Maturation of Rh proteins & --- \\
\hline Pla X & Rhs \& exonemes & --- & Control maturation of SUB1 \\
\hline PLP1 & Micronemes & MACPf pore forming proteins & --- \\
\hline PLP2 & Micronemes & --- & Gametocytes egress \\
\hline ROM1 & Mononemes & Formation of PV & --- \\
\hline SERAs 5 \& 6 & PV & --- & $\begin{array}{l}\text { PV membrane rupture, destabilization of RBC } \\
\text { cytoskeleton }\end{array}$ \\
\hline SUB 1 & Exonemes & Processing of MSP1 and ROM1 & Processing of proteins involved in egress \\
\hline SUB 2 & Micronemes & $\begin{array}{l}\text { Processing of proteases and } \\
\text { regulation of MSPs release }\end{array}$ & --- \\
\hline $\begin{array}{l}\text { CDPK: Ca-dependel } \\
\text { Atypical protein kir } \\
\text { protein; MACPf: Me } \\
\text { anonymous protein } \\
\text { Plasmepsin; PLP: Pe } \\
\text { protease; SERA: Ser }\end{array}$ & $\begin{array}{l}\text { protein kinase; DPAP: D } \\
\text { se; G377: Gametocytes pr } \\
\text { brane attack complex/per } \\
\text { OBs: Osmiophilic bodies, } \\
\text { orin like protein; PV: Paras } \\
\text { e repeat antigen; SUB: Sub }\end{array}$ & $\begin{array}{l}\text { peptidyl aminopeptidase; ECP1: } \\
\text { otein; GEST: Gamete egress and } \\
\text { orin; MSP: Merozoite surface pr } \\
\text { PARPL: Patatin-like phospholi } \\
\text { itophorous vacuole; RBC: Red blo } \\
\text { tilisin-like serine protease. }\end{array}$ & $\begin{array}{l}\text { Egress cysteine protease 1; Fal: Falcipian; FIKK } \\
\text { sporozoite traversal protein; LISP: Liver specific } \\
\text { otein; MTRAP: Merozoite thrombospondin-related } \\
\text { pase; PEG3: Protein of early gametocyte 3, Pla } \\
\text { od corpuscle; Rh: Rhoptry; ROM: Specific rhomboic }\end{array}$ \\
\hline
\end{tabular}


micronemes are the main members for MACPF assigned as pore-forming proteins.

4. Five elements are established as essential components of successful invasion and egress cascade in Plasmodium spp. They are Ca-signaling pathway, as initial stimulus, and apical secretory organelles, PLPs, CDPKs, and different types of proteases, as tools.

5. Micronemes and rhoptries release several molecules required for processing and maturations of the essential proteinases and PLPs. Both PLP1 and PLP2 are involved in merozites and gametocytes egress, respectively.

6.Due to their peripheral localizations, CDPKs are essentially required for the regulation of host cell attachment. Unique protein kinases (FIKKs) are characterized in $P$. falciparum trigging release of $\mathrm{Rh}$ bulb proteins and altering infected RBCs membrane rigidity.

\section{SUGGESTED REFERENCES}

1. Andreadaki M, Hanssen E, Deligianni E, Claudet C, Wengelnik K, Mollard V, et al. Sequential membrane rupture and vesiculation during Plasmodium berghei gametocyte egress from the red blood cell. Sci Rep 2018; 8(1):3543.

2. Azab ME, Abdel Mawla MM. Ultrastructural analysis of posterior polar endocytic phagocytosis by Leishmania amastigotes. PUJ 2011; 4(2): 219-230.

3. Friedrich N, Hagedorn M, Soldati-Favre D, Soldati T. Prison break: pathogens' strategies to egress from host cells. Microbiol Mol Biol Rev 2012; 76(4):707720.
4. Gubbels M-J, Duraisingh MT. Evolution of apicomplexan secretory organelles. Int J Parasitol 2012; 42(12):1071-1081.

5. Guerra AJ, Carruthers VB. Structural features of apicomplexan pore-forming proteins and their roles in parasite cell traversal and egress. Toxins (Basel) 2017; 9(9):265.

6. Hayton $\mathrm{K}$, Templeton TJ. Osmiophilic bodies and the odd organelles of alveolates. Mol Microbiol 2008; 67(2):236-240.

7. Horta MF, Andrade LO, Martins-Duarte ES, CastroGomes T. Cell invasion by intracellular parasites: The many roads to infection. J Cell Sci 2020; 133(4): jcs232488.

8. Ishino T, Tachibana M, Baba M, Iriko H, Tsuboi T, Torii M. Observation of morphological changes of female osmiophilic bodies prior to Plasmodium gametocyte egress from erythrocytes. Mol Biochem Parasitol 2020; 236:111261.

9. Mercier C, Adjogble KD, Daubener W, Delauw MF. Dense granules: are they key organelles to help understand the parasitophorous vacuole of all apicomplexan parasites? Int J Parasitol 2005; 35: 829-849.

10. Mishra M, Singh V, Singh S. Structural insights into key Plasmodium proteases as therapeutic drug targets. Front Microbiol 2019; 10: 394.

11. Sassmannshausen J, Pradel G, Bennink S. Perforin-like proteins of apicomplexan parasites. Front Cell Infect Microbiol 2020; 10: 578883.

12. Singh $P$, Alaganan A, More KR, Lorthiois A, Thiberge $S$, Gorgette 0 , et al. Role of a patatin-like phospholipase in Plasmodium falciparum gametogenesis and malaria transmission. Proc Natl Acad Sci USA 2019; 116(35):17498-17508.

\begin{tabular}{|c|c|c|c|c|c|c|c|c|c|c|}
\hline $\begin{array}{l}\text { Errata } \\
\text { PUJ } 202 \\
\text { Morpho } \\
\text { the first }\end{array}$ & $\begin{array}{l}\text {; } 13(3) \text { : } \\
\text { veek and } k\end{array}$ & $\begin{array}{l}\text { 179-189 } \\
\text { inetics of } \\
\text { posure } t\end{array}$ & $\begin{array}{l}\text { DOI: } 10 \\
\text { susceptil } \\
\text { Schistos }\end{array}$ & $\begin{array}{l}21608 \\
\text { le and } r \\
m a m a\end{array}$ & $\begin{array}{l}\text { puj.202 } \\
\text { esistant } \\
\text { osoni mil }\end{array}$ & $\begin{array}{l}0.47046 \\
\text { Biomphal } \\
\text { acidia }\end{array}$ & $\begin{array}{l}1089 \\
\text { aria alexc }\end{array}$ & ndrina h & emocytes & during \\
\hline $\begin{array}{l}\text { Page 182 } \\
\text { Table 1. Int } \\
\text { periods. }\end{array}$ & roup co & $\bar{~} \overline{\text { arison be }}$ & Ween the & 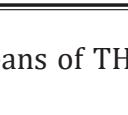 & 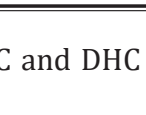 & $\overline{\bar{I}}$ & 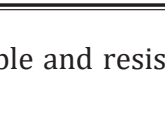 & & & \\
\hline Hemocyte & & & & & Time o & f exposure & & & & \\
\hline Pop \& & Control & (0 hour) & $6 \mathrm{hou}$ & S PO & $1 \mathrm{da}$ & y PO & 3 day & S PO & $7 \mathrm{da}$ & ys PO \\
\hline SubPops & $S$ & $\mathbf{R}$ & $S$ & $\mathbf{R}$ & $S$ & $\mathbf{R}$ & $S$ & $\mathbf{R}$ & $S$ & $\mathbf{R}$ \\
\hline Pop I (B) & & & & & & & & & & \\
\hline & $6.67 \pm 1.63$ & $6.50 \pm 0.84$ & $7.00 \pm 1.67$ & $9.00 \pm 0.63$ & $26.83 \pm 1.60$ & $23.83 \pm 1.33$ & $22.50 \pm 1.97$ & $26.00 \pm 2.20$ & $26.00 \pm 3.03$ & $17.33 \pm 1.51$ \\
\hline GBL & $P=$ & .79* & $P=0$ & & $P=$ & $.01 *$ & $P=$ & & $P=$ & $.00^{*}$ \\
\hline Pop III (G) & & & & & & & & & & \\
\hline TG & $237.33 \pm 6.31$ & $262.83 \pm 5.42$ & $168.83 \pm 4.45$ & $92.5 \pm 1.38$ & $577 \pm 6.81$ & $205 \pm 3.52$ & $573.33 \pm 7.45$ & $792.67 \pm 3.67$ & $583.67 \pm 3.98$ & $640.83 \pm 31.52$ \\
\hline 10 & $P=$ & & $P=0$ & & $P=$ & $.00^{*}$ & $P=0$ & $.00 *$ & $P=$ & $.06 *$ \\
\hline
\end{tabular}

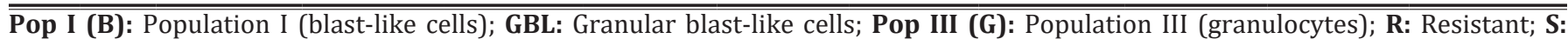
Susceptible; TG: Total granulocytes.

Page 183

Fig. 5. Light micrographs of Giemsa-stained few-granulated granulocytes of B. alexandrina snails: a-d, Adherent cells attached by filopodia (f); e-g, Small granulocytes showing filopodia (f); $\mathbf{h}-\mathbf{j}$, Spindle to oblong few granulated granulocytes with basophilic lobulated nucleus (n), prominent vacuole (v) and few numbers of peripheral basophilic granules (bg). 